\title{
UHX1 and PCTK1: precise characterisation and localisation within a gene-rich region in Xp11.23 and evaluation as candidate genes for retinal diseases mapped to Xp21.1-p11.2
}

\author{
O liver B randau ${ }^{1}$, G erald N yakatura $^{2}$, Kerry B Jedele ${ }^{1}$, M atthias Platzer $^{2}, \mathrm{H}$ elene A chatz ${ }^{1}$, \\ M ark R oss ${ }^{3}$, Jan M urken ${ }^{1}, A$ ndre R osenthal ${ }^{2}$ and $A$ Ifons $M$ eindl $^{1}$

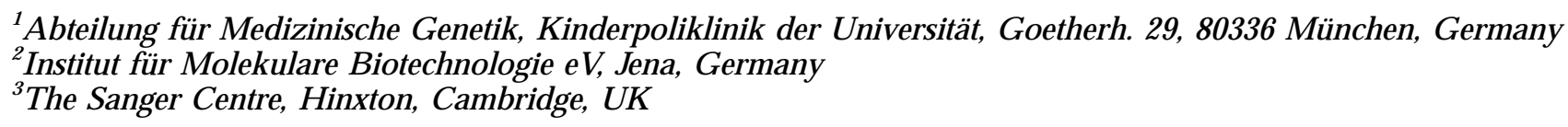

The gene for ubiquitin hydrolase on the $X$ chromosome (UHX1), cloned and mapped to Xp21.2-p11.2, is a candidate gene for retinal diseases. We used fine mapping techniques to localise UHX1 between markers DXS1266 and DXS337, where congenital stationary night blindness (XICSNB) and retinitis pigmentosa type 2 (RP2) are also located. Reevaluation of the UHX1 gene structure demonstrated five new exons, for a total of 21 exons and a predicted protein product of 963 amino acids. Evaluation of patients revealed no UHX1 mutations using SSCP (10 CSNB1 and 20 XLRP) or deletion screening with cDNA hybridisation (13 CSNB1 and 43 XLRP). Likewise, no aberrations were found in the nearby PCTAIRE1 (PCTK1) gene in 13 CSNB1 and 43 XLRP patients by deletion screening. Thus mutations of $U H X 1$, and probably PCTK1, do not appear to cause common X-linked eye diseases. UHX1's role in patients with mental retardation may be appropriate for further investigations into UHX1 function.

Keywords: UHX1; PCTK1; retinal disease; mental retardation

\section{Introduction}

U biquitin hydrolases are a group of enzymes found in multiple tissues and involved in regulation of cellular protein function through cleavage of ubiquitin from its conjugated forms. These enzymes thus play an impor-

Correspondence: $\mathrm{Dr}$ A Ifons Meindl, A bteilung für Medizinische Genetik, G oethestrasse 29, 80336 M ünchen, G ermany. Tel: +49 895160 4467; fax: +49 895160 4780; E mail: alfons@pedgen.med.uni-muenchen.de

R eceived 10 N ovember 97; revised 2 February 98; accepted 18 February 98 tant role in protein degradation and reversible protein modification. A novel gene encoding a ubiquitin $C$-terminal hydrolase on the $X$ chromosome (UHX 1 ) has recently been described. ${ }^{1} A$ widespread tissue expression with a five- to ten-fold higher expression in the retina was shown. Because of this high retinal expression and evidence suggesting abnormalities in protein processing as the etiology of several retinal diseases, ${ }^{1} \mathrm{UHX} 1$ was proposed as a candidate gene for retinal diseases. A nother gene in this region, PCTAIRE-1 (PCTK1), is a member of PCTAIRE, a subfamily of the cdc2-related serine-threonine specific 
protein kinase family and involved in cell cycle regulation. ${ }^{2} \mathrm{UHX} 1$, ubiquitin activating enzyme E 1 (UBE 1) and $\mathrm{PCTK} 1^{3}$ have all previously been mapped to $X$ p21.2-p11.2, a region known to contain several retinal diseases, including $X$-linked congenital stationary night blindness, ${ }^{4-7} X$-linked retinitis pigmentosa types 2 and 3 $(\mathrm{RP} 2, \quad \mathrm{RP} 3)^{8-11}$ and $X$-linked cone dystrophy $(X L C O D){ }^{12}$ (for review see $R$ osenfeld et al. ${ }^{13}$ ).

In addition to $\mathrm{UHX} \mathrm{I}^{\prime} \mathrm{s}$ possible retinal function, recent data suggest a role for ubiquitin $\mathrm{C}$-terminal hydrolase in long-term facilitation in A plysia. ${ }^{14}$ This investigation into learning and memory pathways in a mollusk model might carry important implications for causation of mental retardation, learning disorders and memory disturbances in humans. Interestingly, several $X$-linked mental retardation families also map to this region of the $X$ chromosome (for review see $L$ ubs et al. ${ }^{15}$ ) including one family with XLMR and XLRP. ${ }^{16}$ Thus the actual function(s) of UHX 1 in neurologic and possibly other pathways is at present unclear.

O ur investigation explores several aspects of UHX 1 and PCTK 1 gene structure and function. The possible role of $\mathrm{UHX} 1$ involvement in learning and memory is still preliminary and beyond the scope of this investigation. We concentrated instead on the possible causation of retinal disease by $U H X 1$ and PCTK 1 . First, we performed fine mapping of these two genes to determine exactly which of the retinal diseases localised to this region of the $X$ chromosome might be caused by UHX 1 or PCTK 1 mutations. Next, determination of the precise genomic structure was done to allow accurate identification of gene defects. Finally, mutation screening was performed to examine the possible causative role of UHX 1 and PCTK 1 in CSNB 1 or R P2, the retinal diseases located in the same region.

\section{Materials and Methods}

\section{Probes and Hybridisation}

A probe for the ZNF 157 gene $^{17}$ was obtained with primers published by $\mathrm{C}$ arrel et al. ${ }^{3}$ The $5^{\prime}-\mathrm{UHX} 1$ probe was derived with nested primers: XIF: AAGAAAGATGGCACTTGGCCC, $\quad X 1 R$ : $\quad$ TCGTCCTCATCATCTGAGTTGGG, X laF: ACA CTGCATGTCATGAA CA A , $X$ laR: TGGTCACGTAGCGTGAGAGC. The 3'UHX 1 probe was derived with the following nested primers: $X 2 F$ : A T G CTTTTTG GACA C CCCCTC, X2R: ATCCATG A ACTCAGAGCTGGG，X2aF： A CCGCTTCACCTGGGAGGG, X 2aR: AGCTGCAGGCAGGGGAGGC. The PCTK 1 probes were derived with the following nested primer pairs: Pct1F: ATGAAGAAGATCAAACGGCAGC, Pct1R: TGTGGCATCCCTCCA GCCG, Pct2F: ATAGACAAGACCAATGGTGCC, PCt2R: TCTGTGGCTTA -
GAACTCGGTG. A probe for the DXS8237E gene was produced using the primer pairs: $F 2$ : CCAGTCTCG GACTTGGTTG, R 4: AGGCTGGGGGCGAGAGAAAG ; F3: GGCTGAGCTGGG AGAGTTGG, R 5: TGCATCGA CACA GAGTCGG. The NCBI clones R 41358 and D 86969 were kindly provided by Reference Library Data $B$ ase (RLDB), Berlin-Charlottenberg: ${ }^{18}$ inserts from both clones were obtained by using vector-specific primers. H ybridisation of patient DNA was carried out in Church buffer with stringent washing conditions $(0.1 \times \mathrm{SSC})$.

\section{Mapping Panels and PACs}

R adiation-induced somatic cell hybrids A 19D 9 and A 19E 8 were generated as described. ${ }^{19}$ Polymorphic microsatellites used for characterisation are contained in the $G$ énéthon human linkage map. ${ }^{20}$ The ICR F- and CEPH YACs used as mapping resources (Figure 1 ) were obtained from RLDB, Berlin. ${ }^{18}$ PACs containing the markers DXS1264, DX S1003, D X S1266, D X S337, and E LK 1 were isolated from the deJ ong PAC library by the Sanger Centre (http://www.sanger.ac.uk/ HEP/Chr.X/; X ctg311, X ctg448, X ctg597) and distributed by RLDB, Berlin; PACs containing DXS8237E and/or UHX 1 were isolated by hybridisation from the dej ong PAC library and provided by RLDB, Berlin.

\section{Identification and Analysis of Genomic Clones}

The Lawrence Livermore $X$ cosmid library distributed by $R L D B, B$ erlin, was hybridised with a partial UHX 1 CDNA. $\mathrm{H}$ ybridisation with the $5^{\prime}$ and $3^{\prime}$ parts of the published $\mathrm{UHX} 1$ CDNA revealed a complete representation of $U H X 1$ on cosmid L L N L C110E 2210 Q. This cosmid was sequenced after subcloning in $\mathrm{pUC}$ vectors. The $\mathrm{pUC}$ clones were shotgunsequenced using dye-terminator chemistry. The sequences were assembled and edited with the X G A P program. ${ }^{21} \mathrm{G}$ aps were closed using custom-made primers on pU C templates, PCR-products or cosmid DNA. The finished sequence revealed a contig of 41536 base pairs (G D B acc. no. U 62534). Exon-intron boundaries were obtained by comparing the genomic sequence with the published CD NA sequence and by exon-prediction programs MC-VECTOR, FEXH AND XGRAIL $1 \mathrm{a} / 2$.

\section{PCR Reactions and SSCP Analysis}

A nnealing temperatures for the primer pairs are listed in Table 1. A II 21 exons of the UXH 1 gene were amplified under the following conditions: after an initial denaturation for $5 \mathrm{~min}$ at $94^{\circ} \mathrm{C}$, denaturation was at $94^{\circ} \mathrm{C}$ for $1 \mathrm{~min}$, annealing at the exon-specific temperature for $1 \mathrm{~min}$ and extension at $72^{\circ} \mathrm{C}$ for 35 cycles. A mplified fragments from all exons were analysed by SSC $\mathrm{P}^{22}$ using Hydrolink ${ }^{\mathrm{TM}}$ (AT Biochem., Malvern, PA, USA) or SERDO-gels (B oehringer-Ingelheim Bioproducts Partnership, Heidelberg). Staining was performed with Sybrgreen (M olecular-Probes E urope BV, L eiden, The Netherlands), and band visualisation was done with Fluorlmager (Molecular Dynamics G mbH, Krefeld, Germany), both according to the manufacturer's recommendations.

\section{DNA samples}

DNA was extracted from peripheral blood samples from unrelated patients with $X$-linked retinal diseases: 43 affected individuals from R P families without RPG R gene mutations (phenotypes as described by $\mathrm{M}$ eindl et al., ${ }^{23}$ and 13 affected 


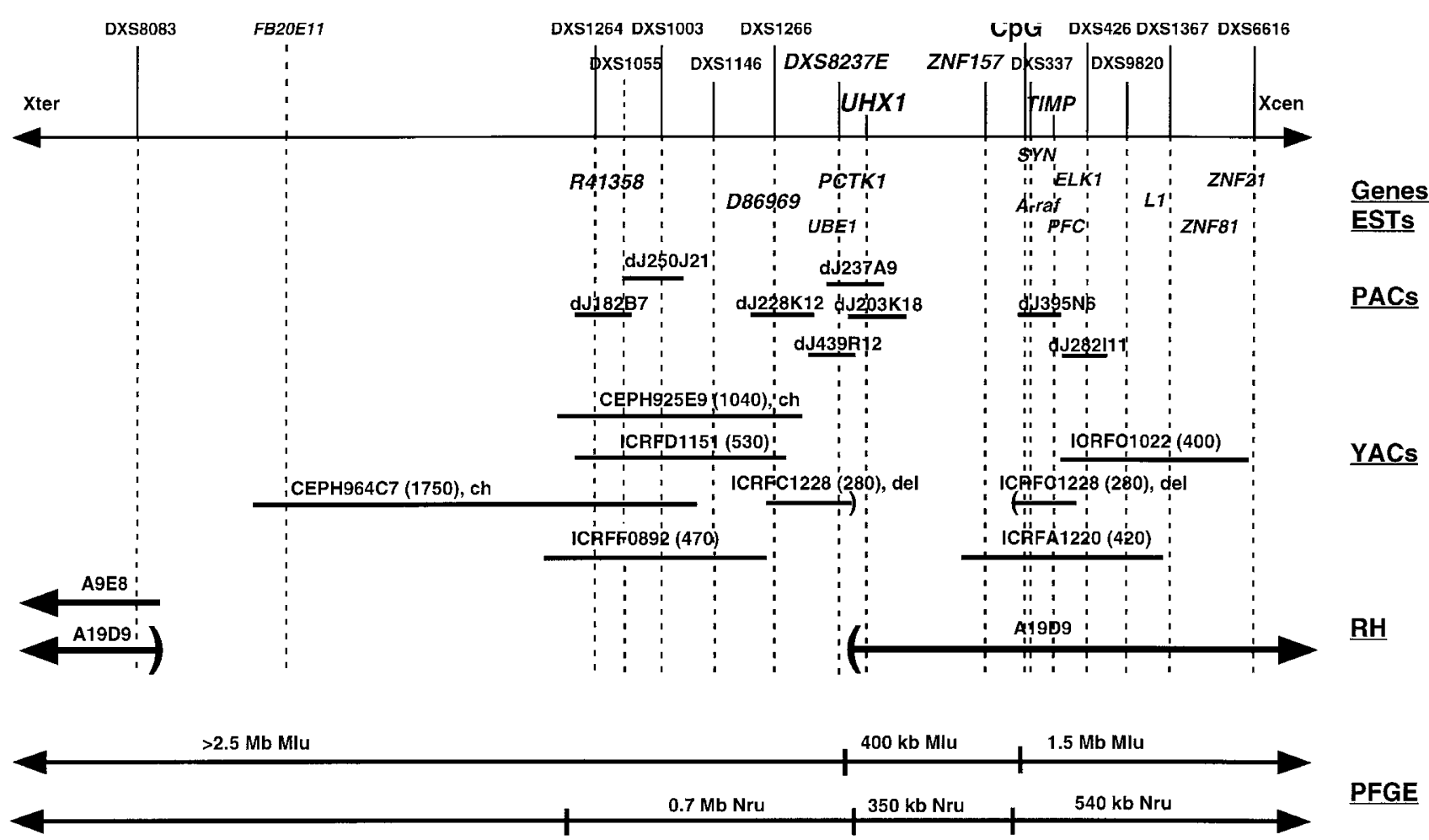

Figure 1 Fine mapping of UHX 1 between DXS1266 and DXS337. (a) A schematic representation of a physical map between D X S8083 and DX S6616 is shown. A 19E 8 and A 19D $9(\mathrm{RH})$ are radiation hybrids, ${ }^{19} \mathrm{PAC}$ from the $\mathrm{P}$ dej ong library (RPCI - 1 and 3) were obtained either from Sanger Centre or RL DB, B erlin; YACs (sizes in brackets) were either from the ICRF or CE PH libraries (http://www.ceph.fr/cgi-bin/http_infoclones) and provided by RLDB, Berlin. R41368 and D 86969 are NCBI ESTs initially mapped between D X S1201 and D X S1039 (http://www.ncbi.nlm.nih.gov/cgi-bin/ SCIE N CE 96). D NA markers given above are published. ${ }^{26,27}$ Deletion of ICRF YAC y900C 1228 was shown by PFGE analysis.

individuals from $X$-linked CSN B families (E Zrenner, unpublished data, 1997). The 43R P families contained only a single family identified as recombinant with the R P 3 interval, whilst the remaining 42 could be either R P2 or R P3 families based on linkage studies.

\section{Results}

\section{Fine Mapping of UHX1 Between the Markers DXS1266 and DXS337}

A CDNA probe encompassing the 3 ' part of the published UHX 1 sequence was used to hybridise D NA S from different radiation hybrids, ${ }^{19}$ each maintaining different portions of Xp11.4-Xp11.22. UHX1 was present on radiation hybrid $A$ 19D 9, while probes for DXS8237E, ${ }^{24}$, D 86969 and R 41358 (NCBI ESTs located between the markers DXS1201 and DXS1039) demonstrated no hybridisation signal. The proximal localisation of $\mathrm{UHX} 1$ was further confirmed by PFGE analysis, which showed the UHX 1 gene together with the ZNF 157 gene $^{17}$ on a $350 \mathrm{~kb}$ N rul fragment, whilst
DX S8237E and D 86969 were located on a $700 \mathrm{~kb} \mathrm{~N} \mathrm{rul}$ fragment. DX S8237E and UHX 1 also segregated with different Mlul fragments (Figure 1 and Figure 2). The NCBI EST R 41358 was mapped to the PAC dJ 182B 7, which is part of a contig around DXS1055 and DXS1003. It also identified the $700 \mathrm{~kb}$ Nrul fragment and $3 \mathrm{M} \mathrm{b}$ M lul fragment in genomic D NA, but was not present in a constructed PAC contig encompassing the region between the markers DXS1266 and UHX 1 . Thus, DX S1055 and DX S1003 were clearly distal to the marker DX S1266. The IMAGE clone D 86869, which also identified the large M lul fragment and the $700 \mathrm{~kb}$ $\mathrm{N}$ rul fragment, was mapped to the PAC dJ 228K 12 , suggesting a localisation of this EST between DX S1003 and DXS8237E. PACs containing the markers DXS1264 (d) 182B 7), DXS1003 (dJ 250) 21), DXS1266 (d) 228K 12), DX S337 (dJ 395N 6) and E LK 1 (dJ 282I 11) were identified using the A cedb software of Sanger Centre ( $X$ chromosome status map: $45000 \mathrm{~kb}-47000 \mathrm{~kb}) ;$ PACs containing the genes DX S8237E, PCTK 1 and/or UHX 1 were isolated from 
Table 1 Exon-intron organisation of the UHX 1 gene

\begin{tabular}{|c|c|c|c|c|c|}
\hline Exon & Position & $\begin{array}{l}\text { Exon } \\
\text { size }\end{array}$ & Splice acceptor & Splice donor & $\begin{array}{l}\text { Intron } \\
\text { size }\end{array}$ \\
\hline 1 & $1-439$ & 439 & CTTTCCATGC & GCGA A A GCT G gtgaggctgggctgc & 5852 \\
\hline 2 & $440-549$ & 109 & ctgtctgggccccagG TTCCTT G TG & СТCTTTCA A G gtacaaggcctttgc & 173 \\
\hline 3 & $550-680$ & 130 & acccccgccccacagA TG A G A TA A A & T GA A C G CA A G qtataatqqatgqqq & 309 \\
\hline 4 & $681-798$ & 117 & cctctactcttacagG TCA TA G A G C & G ATT CTA TT G gtgagtctaagggtc & 398 \\
\hline 5 & 799-944 & 195 & catcaccccgcacagG CCTA G TA TT & G A CTG G G CA G gtaagggtggggagg & 137 \\
\hline 6 & $945-1006$ & 61 & ctttctccaacccagTTG A T CA T CA & ZG CATGTCA T gtgagcccttggggt & 126 \\
\hline 7 & 1007-1109 & 102 & ctcatctaaccccagG A A CA A CA A C & G G G C C CT G CA G gttgggccattatag & 401 \\
\hline 8 & $1110-1283$ & 173 & ctctggcctctgcagT G CCTCA G CA & T G T G TTCA A G gtgtgactcaaccct & 91 \\
\hline 9 & $1284-1442$ & 158 & tgcccatcttcttagA A CA A G G TT G & A C C G G A TCA G qtaggctgccccac & 383 \\
\hline 10 & $1443-1667$ & 224 & tgacactatcaacag G A G G TG G CA C & G CCA G A G CA G qtatgagqcagtgqu & 133 \\
\hline 11 & $1668-1751$ & 83 & ccgttttcccttagCA CC G G CTCG & G CCA G A G A G G gtgagactgcagaag & 104 \\
\hline 12 & $1752-1846$ & 94 & atatcccattcctagA T G A T G G T G G & A TA TCTTCG T gtgagtggggatggC & 676 \\
\hline 13 & $1847-2053$ & 206 & gtccaccccccacagC TA T G A G G TG & A C CG G CT CT C gtaagtgtcctcttc & 897 \\
\hline 14 & 2054-2106 & 62 & tctgctctgttgcagA C G CTA C G T G & G A T G A G A A A G gtgagggggctaaca & 132 \\
\hline 15 & 2107-2349 & 242 & atcctcctgtcctagA A G A T G A CG A & G A A G T CCA T G gtatttcctttggct & 92 \\
\hline 16 & $2350-2420$ & 70 & ctccctcacccccagC C CA G C CG TA & A G A G G CTG A G gtaaatgagatccca & 284 \\
\hline 17 & $2421-2533$ & 112 & ttcacccaatcctagG G CTA CG T G A & A A A A C C C CT G gtgaggggccagagc & 1590 \\
\hline 18 & $2534-2683$ & 149 & gggctctgtctgtagG TA CT G C C CT & TTC CTA T C CG gtcaggggccaggga & 83 \\
\hline 19 & 2684-2799 & 115 & tctccccacccacagG G A C CT G G A C & GATG GA CA CT gtatgtccaggctg & 192 \\
\hline 20 & $2800-2888$ & 88 & ctccttccctttcagA CA CA A CA TT & TCA G A T C G A G gtgtgacttccatcc & 94 \\
\hline 21 & $2889-3423$ & 534 & gtcctctccccacagT C CA A G G CA G & TCTGCTCCCC & \\
\hline
\end{tabular}

the dej ong PA C library and provided by R LDB, Berlin (see Figure 1: dJ 439R 12, dJ 237A 9, dJ 203K 18).

The new localisation of UHX 1 between DXS1266 and DX S337 in X p11.23 excluded it as a candidate gene for RP3 and XLCOD, and narrowed the field of possibly-related retinal diseases to RP2 and CSN B 1. ${ }^{4,6,25}$

\section{Genomic structure of the UHX1 gene}

Close linkage of UHX 1 to DX S8237E and PCTK 1 was shown by the hybridisation of these genes to the PAC d) 237A 9. A II genes were found on this PA $C$, while PA C d) 203K 18 contained only UHX 1 and PCTK 1 , indicating a small distance between these two genes (Figure 1 ). To determine the genomic structure of the $\mathrm{UHX} 1$ gene, two UHX 1 cosmids from the Lawrence Livermore library ( $L L X N 01)$ were isolated. Both cosmids were shown by PCR also to contain the PCTK 1 gene which maps close to the ubiquitin activating enzyme $E 1$ gene (UBE 1). UHX 1 was originally described as a $3121 \mathrm{bp}$ DNA sequence with a $2070 \mathrm{bp}$ open reading frame (ORF), coding for a 690 amino acid sequence ( 1 , A cc.N 0.:H SU 44839). In this report, the ORF was preceded by a 679 bp 5' untranslated region (UTR) and a 410 bp 3'UTR containing an atypical polyadenylation signal and ending with nine adenines. $O$ ur data indicate that the previously published CDNA needs to be adjusted at the following positions: $\mathrm{G}$ at nucleotide 35 to $A, G$ at nucleotide 47 to $C$, delete $G$ at nucleotide 55 , $G C$ at nucleotides 111 and 112 to $C G$, insert $G$ at nucleotide 212 , insert $G$ at nucleotide 262 , insert $G$ at nucleotide 292 , insert $G$ at nucleotide 302 , and $G C$ at nucleotides 342 and 343 to CG. These inaccuracies led to the prediction of a long $5^{\prime} U T R$ and an altered OR F designation. We amplified our own CDNA sequence isolated from brain (GIBCO) encompassing the entire $5^{\prime} U T R$, and reanalysed it for intron-exon boundaries using cosmid sequencing and computer analysis (Table2, Figure 3). Five previously undescribed exons were thereby identified, extending in the $5^{\prime}$ direction. The new findings showed that the $O R F$ frame consisted of $3023 \mathrm{bp}$ with a total coding region of $2889 \mathrm{bp}$ and a 5'UTR $134 \mathrm{bp}$ long. All exons, spread over $16 \mathrm{~kb}$ (Figure 3a), could be identified by the gene structure programs XGRA IL 2 and FEXH, except exon 1. Exon 1 was recognised by GRA IL 1a, although this program did not identify the exon-intron boundaries of the predicted exons accurately. No promoter region could be detected by either XGRAIL 2 or PROMOTER SCA N II (Figure $3 b$ ).

\section{Genomic Structure of the PCTK1 gene}

Full sequencing of the UHX1-containing cosmid revealed a second gene close to the UHXI gene (Figure 3). This gene consists of at least 15 exons and a 


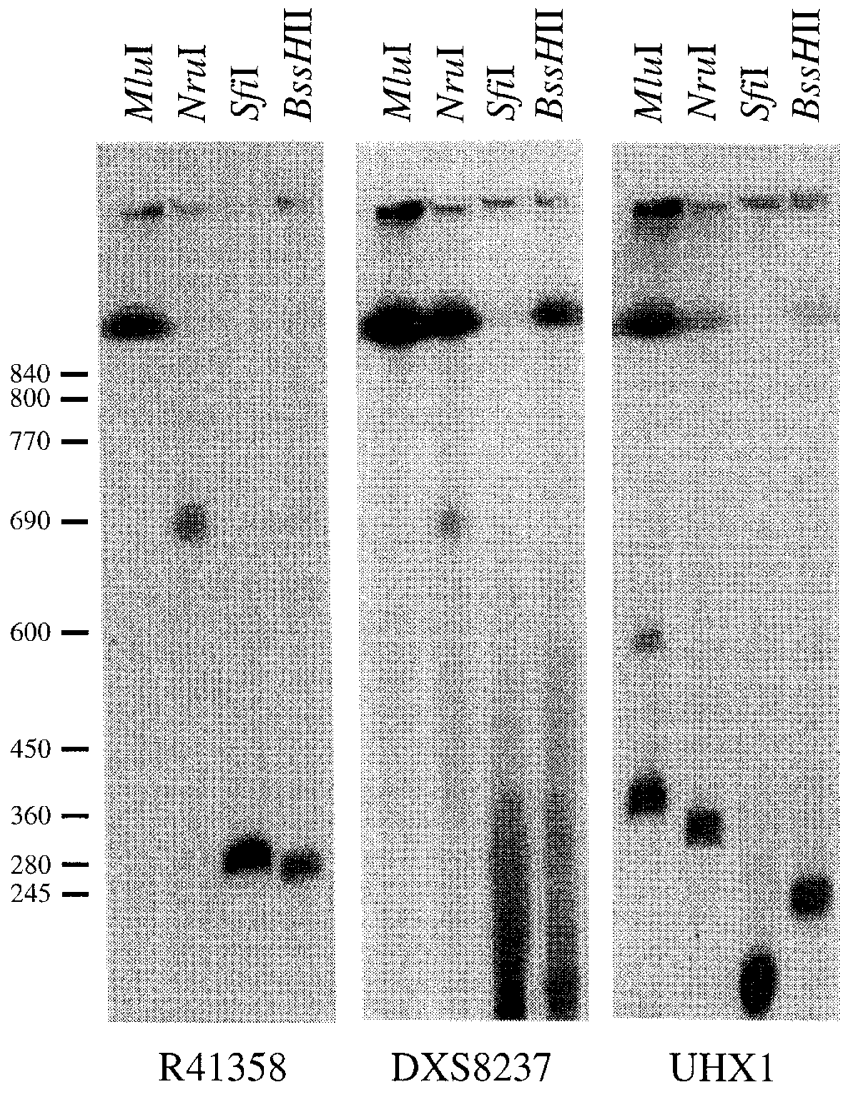

Figure 2 Mapping of UHX1, DXS8237E and R41538 by PFGE analysis. Hybridization was performed under stringent conditions. Sizes are indicated on the left. $\mathrm{H}$ igh resolution PFGE indicates a size for the Mlul fragment identified by DXS8237E and R41358 of $>2.5 \mathrm{Mb}$. PFGE blots were prepared as described in Schindelhauer et al. ${ }^{27}$ homology search demonstrated identity with the previously published PCTK 1 CD NA. ${ }^{3}$ Exon-intron boundaries of the 15 exons are described in Table 2. A gain all exons, except exon 2 and 5 , could be predicted by FEXH and XGRA IL 1a/2 computer analysis. No promoter region was identified, probably due to insufficient sequence data in the $5^{\prime}$ direction (Figure 3a). A combination of our data with that of Carrel et $\mathrm{al}^{3}$ indicates that the UHX 1 gene is located $17 \mathrm{~kb}$ from the UBE 1 gene.

\section{Mutation Analysis of UHX1 and PCTK1 in Patients with Retinal Disorders}

To examine the possible causative role of UHX 1 in retinal diseases mapped to Xp21.2-p11.2, DNA samples from patients with X LR P (43 cases) and X LCSN B (13 cases) were digested with EcoRI and Pstl and hybridised with the UHX 1 CDNA probe. From the 43 $X L R P$ families linked to the $R P 3$ and $R P 2$ region, only $30-40 \%$ can be expected to be of the R P2 type, even after exclusion of RPG R mutations.

No aberrations of the UHX 1 gene were detected in any of the patient samples using this technique. $\mathrm{H}$ ybridisation revealed one fragment of $9 \mathrm{~kb}$ with the $5^{\prime}$ probe and two fragments of 3 and $12 \mathrm{~kb}$ with the $3^{\prime}$ probe. In 20 of the 43 XLRP and 10 of the 13 XLCSXIB patients, all 21 exons from the UHX 1 gene were amplified with 18 primer pairs (Table 3 ). No sequence changes could be detected in any patient analysed by SSCP analysis. A II 43 XLRP and 13 XLCSNB patients were also screened for intragenic deletions in the PCTK 1 gene using a CDNA probe. A single $20 \mathrm{~kb}$ $E c o R I$ fragment and two Pstl fragments of $2 \mathrm{~kb}$ and

Table 2 Exon-intron organisation of the PCTK 1 gene

\begin{tabular}{|c|c|c|c|c|c|}
\hline Exon & Position & $\begin{array}{l}\text { Exon } \\
\text { size }\end{array}$ & Splice acceptor & Splice donor & $\begin{array}{l}\text { Intron } \\
\text { size }\end{array}$ \\
\hline 1 & $1-325$ & 325 & TTCCATCGCT & TCTG CA CCA G gtgggtccactggct & 626 \\
\hline 2 & $326-459$ & 133 & tctccctgccacaagA G ATTGTGCA & CT C CA CT G A G gtgcttgaccccgtc & 100 \\
\hline 3 & $460-585$ & 125 & tgccttacctgctagG A CA T CA A CA & T GT CA G C CTA gtaagcaccttctgt & 91 \\
\hline 4 & $586-642$ & 56 & tcatttgtcccacagT CTG A G ATT G & A CT G G G C G A G gtgagaggcaaatag & 98 \\
\hline 5 & $643-757$ & 114 & ttcctcacattccagG G TTA C CTA T & A TCCG G G A A G gtacacacccccatc & 670 \\
\hline 6 & $758-852$ & 94 & ccatgcttcctgcagT G T C C C T G C T & T G A G TA C CT G gtaaggttgagtggc & 99 \\
\hline 7 & $853-915$ & 62 & ctcttctttcctcagG A CA A G G A CC & CA A C GTG A A A gtgggtgtggggcag & 252 \\
\hline 8 & 916-1039 & 123 & ttcctgggtccccagCTG TTCCTG T & G CT G A CTTT G gtaccactggcctcc & 181 \\
\hline 9 & $1040-1160$ & 120 & ttctctgatttccagG C C T G G C C C G & TTG A CA T G T G gtaaggacaggtgga & 109 \\
\hline 10 & $1161-1258$ & 97 & gccctccctgcccagG G G T G T G G G C & CG TATCTTA G gtgaggaggcatggg & 85 \\
\hline 11 & $1259-1364$ & 105 & ctttttccctactagG A A C C C CA A C & ACGCA CCCCG gtgaggatggtgggt & 82 \\
\hline 12 & $1365-1407$ & 42 & ttgtccttgtggcagA C TT G A TA G C & G CT G TTG CA G gtgagaccaccttgg & 156 \\
\hline 13 & 1408-1498 & 90 & cgcacccccactcagT TT G A G G G TC & СTTCCTG A CA gtgagtggagctggg & 1098 \\
\hline 14 & $1499-1576$ & 77 & ctcccccatctgtagC TA СТ TC CA T & CCTG A CT CA G gtaggtatagcccct & 87 \\
\hline 15 & 1577-1745 & 168 & tgctttcccccacagG CA G G C CA G C & CCACTTGTCC & \\
\hline
\end{tabular}


$3.7 \mathrm{~kb}$ could be detected with the full-length cDNA . This is in agreement with the partial restriction map for PCTK 1 previously published. ${ }^{3} \mathrm{No}$ deletions or R FLPS could be found in $43 R P$ and $13 X$ LCSN B patients from unrelated families. Since deletion screening and SSCP have only a limited sensitivity, we cannot fully exclude mutations in the UHXI and PCTK1 gene in the analysed patients.

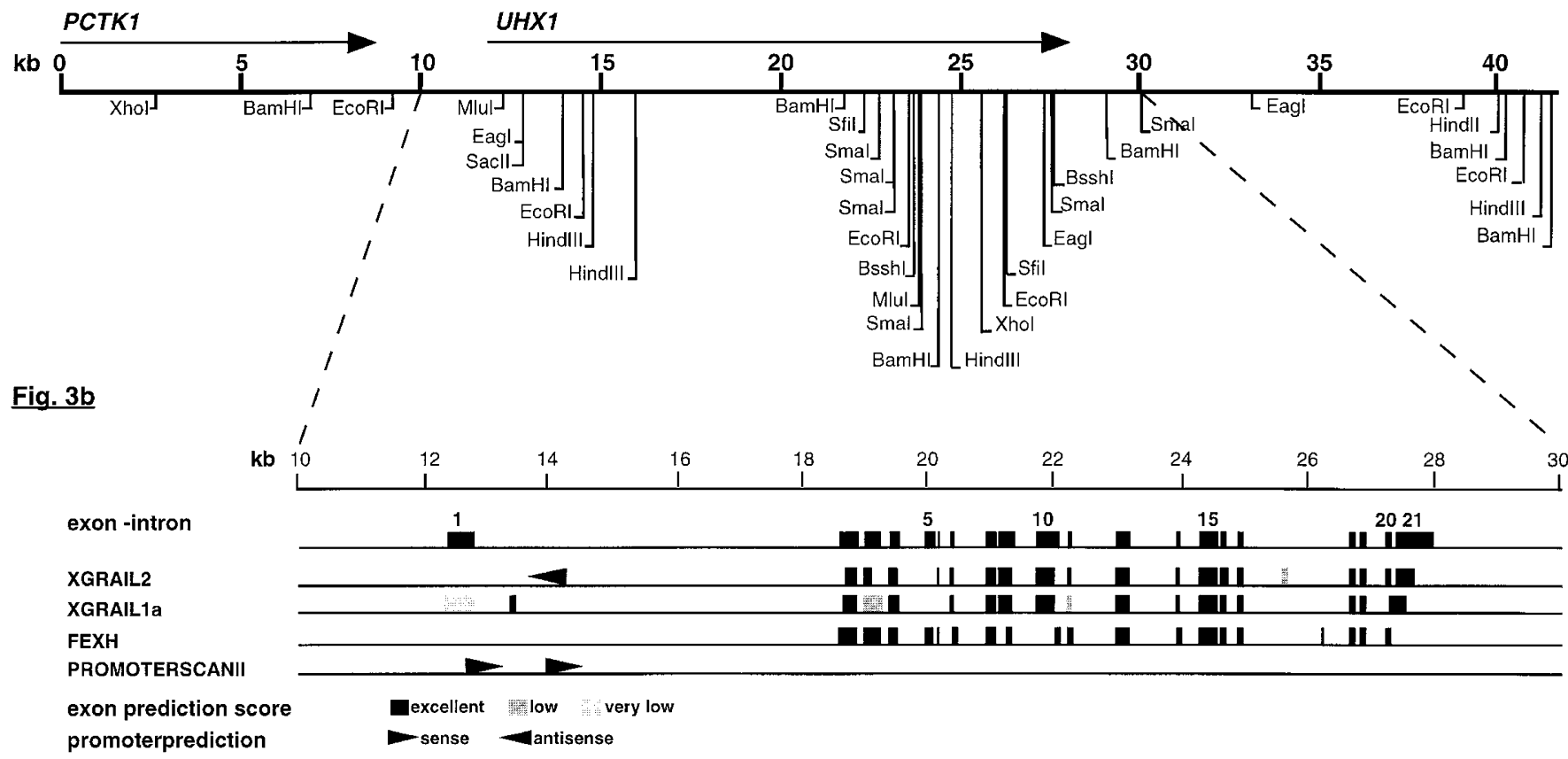

Figure 3 (a) Sequence based restriction map of cosmid E $2210 Q$ containing the genes for PCTK 1 and UXH 1. (b) UHX 1 exons and results of computer-generated intron-exon boundary determinations. $E$ xons are represented by black boxes. Predicted excellent exons by the used gene structure programs are indicated by black boxes, low and very low predicted exons are shaded grey. Promoter motifs are shown by triangles.

Table 3 SSCP primers for UHX 1 screening

\begin{tabular}{llll}
\hline Exon & Forward sequence & Reverse sequence & A nnealing T ${ }^{\circ} \mathrm{C}$ \\
\hline 1 & TGTGCACGGGGCCATTTC & ACCGCAGCCCAGCCTCACC & 60 \\
2 & TATAGCTAACCTCAGCTACC & AAGAACTCCAGGGCTAGG & 51 \\
3 & TTCTTCCTCTGCCACCTCC & TTTCTTACTACGCACTCCCC & 51 \\
4 & ATAGCTAACCCTCAGCTACC & AAGAACTCCAGGGCTAGGG & 55 \\
5 & TCTCGAATGTACTCCATCACC & TGAACAGAAAAGCCCTCCCC & 55 \\
$6 / 7$ & AACCCAGTTGATCATCATGG & AGACACAACTATAATGGCCC & 55 \\
8 & TCTGCCTCATTCACCTGGTC & AGAGCAAGAGACGTAGGG & 60 \\
9 & TCTGATGACCCTGCCCATC & TTGGTGATTTAATGGACTGC & 55 \\
10 & TCTTACCTGGGCAAGCCC & ACCAAGTTCCTGAGGAACCAC & 55 \\
$11 / 12$ & TTTGTGGTACCGTTTTCCC & AAGTCGGAGTTCAGACCCC & 55 \\
13 & TGATCAGGTGTGTGCCTGCTGTC & ACCTAACCCAGAGCCCC & 55 \\
14 & ATCTTAATAAAATCCCGGC & AGCCCTCGCTGATAGCCAAAGG & 55 \\
15 & TCCCATCTCTGACATCCTCCTG & AAGAGCTGGAAGTACCCC & 55 \\
16 & ATGACCACCTCTCCCTCAC & TGAAACCCCCACACACAGG & 55 \\
17 & ATCTGGTTGTCTGTCACCC & ATCCTCTCCCTGGCCCC & 55 \\
18 & AAGTCCGTTTGCTGACTCGG & AGCCTGGCACATACAGTGTCC & 54 \\
$20 / 21$ & TACCAGTATTAACCCTCTC & TTTCTGTGGCAGGACCCAG & 57 \\
\hline
\end{tabular}




\section{Discussion}

Precise knowledge of the fine mapping and genomic organization of the UHX 1 gene is necessary to allow investigation into its function. Complete sequencing of a cosmid ( $L L N L C 110 E 2210 Q$ ) containing the entire UHX 1 gene revealed differences in the CDNA that shortened the open reading frame in the original study. $G$ enomic sequencing revealed five more exons in the $5^{\prime}$ sequence, yielding a gene of about $16 \mathrm{~kb}$ with 21 exons. The predicted ubiquitin-related enzymatic function was not altered.

The second gene found on the cosmid, PCTK 1 , has already been cloned $^{1}$ and mapped adjacent to UBE $1 .^{3}$ PCTK 1 belongs to the cdc2-related protein family, which is involved in the regulation of mitosis. ${ }^{2}$ So far, no diseases associated with mutations of the PCTAIRE subfamily have been described.

Comparison of the mapping databases did not allow construction of a consensus map for the region around UXH 1. Preliminary PFGE mapping experiments indicated UHX 1 as the most centromeric marker, followed by PCTK 1 and UBE1. Two new findings helped determine the orientation of the markers: the location of markers DXS1003 and DXS1055 telomeric to UXH 1 and DXS8237E, and UHX1's centromeric position relative to $D X S 8237 E$. The proximity of UHX1 to PCTK 1 , and TIMP1 to ARAF 1 suggested a different orientation from that published by Carrel et $\mathrm{al}^{3}{ }^{3}$ possibly because their study used a YAC (ICRF-YAC y900C 1228$)^{26}$ since shown to be deleted [own data].

The localisation of UHX 1 and PCTK 1 limited their candidacy to RP2 and CSNB 1 and excluded RP3 and $X L C O D$. No aberrations could be detected for UHX 1 by SSCP analysis, or by deletion screening for UHX 1 and PCTK 1. Evaluation for point mutations in PCTK 1 is underway. Thus, $U H X 1$, and to a lesser extent PCTK 1, are unlikely to cause retinal diseases. We did not exclude other mechanisms by which retinal diseases might still be caused by these two genes, such as the possibilities of either mutations in the as yet unidentified promoter regions for the two genes or another retinal disease mapping to Xp21.2-p11.2. In addition, the finding of four different genes (UHX1, PCTK 1 , UBE 1 and EST DXS8237E) within $70 \mathrm{~kb}$ indicates a gene-rich region, leaving a number of candidate genes for R P2 and CSNB 1 to be explored.

$A$ recent report by $\mathrm{Hegde}$ et $\mathrm{al}^{14}$ indicates that ubiquitin $\mathrm{C}$-terminal hydrolase is essential for longterm facilitation in the mollusc Aplysia. Therefore, UHX1 might be a candidate for investigations of human learning and memory, especially syndromal and non-specific $X$-linked mental retardation (XLMR) already mapped to this region. ${ }^{15,16}$ The role of $U H X 1$ in the regulation of normal growth and cancer development, suggested by Swanson et $\mathrm{al}^{1}{ }^{1}$ may also be appropriate for future studies.

In summary, we present important new data which enabled the precise genetic structure of UHX 1 , and the fine mapping, which is an important prerequisite to establish a sequence ready map for the R P2 region. A bnormalities in UHX 1 and PCTK 1 are unlikely to be the cause of XLRP2 or XLCSNB. Other possible functions of UHX 1 in memory, learning, and normal and neoplastic growth remain to be explored.

\section{Acknowledgements}

We thank Drs B Wittwer, B Lorenz and E Zrenner for providing blood samples from XLRP and XLCSNB patients and $\mathrm{H} \mathrm{H}$ ellebrand. This work was supported by a BMBF grant for 0 Brandau.

\section{References}

1 Swanson DA, Freund CL, Ploder L, M cl nnes R R, Valle D: A ubiquitin $C$-terminal hydrolase gene on the proximal short arm of the $X$ chromosome: implications for $X$-linked retinal disorders. Hum M ol G enet 1996; 5: 533-538.

2 Okuda T, Valentine VA, Shapiro DN, Downing JR: Cloning of genomic loci and chromosomal localization of the human PCTAIRE -1 and -3 protein kinase genes. G enomics 1994; 21: 217-221.

3 Carrel L, Clemson CM, Dunn JM et al: $X$ inactivation analysis and DNA methylation studies of the ubiquitin activating enzyme E 1 and PCTAIRE-1 genes in human and mouse. $\mathrm{H}$ um $\mathrm{M}$ ol $\mathrm{G}$ enet 1996; 5: 391-401.

4 M usarella MA, Weleber R G, M urphey WH et al: A ssignment of the gene for complete $X$-linked congenital stationary night blindness (CSNB 1) to chromosome $X$ p11.3. G enomics 1989; 5: 727-737.

5 A Idred M A, D ry KL, Sharp D M et al: Linkage analysis in $X$-linked congenital stationary night blindness. $G$ enomics 1992; 14: 99-104.

6 Bech-Hansen NT, Moore BJ, Pearce WG: Mapping of locus for $X$-linked congenital stationary night blindness (CSNB1) proximal to DXS7. Genomics 1992; 12: 409-411.

7 Dry $K L$, Van LL, A Idred MA, Brown J, Hardwick JJ, Wright A F : Linkage analysis in a family with complete type congenital stationary night blindness with and without myopia. Clin G enet 1993; 43: 250-254.

8 Coleman M, B hattacharya S, Lindsay S et al: L ocalization of the microsatellite probe DXS426 between DXS7 and $D X S 255$ on $X p$ and linkage to $X$-linked retinitis pigmentosa. A m J H um G enet 1990; 47: 935-940. 
9 Wright A F, B hattacharya SS, A Idred MA et al: Genetic localisation of the RP2 type of $X$ linked retinitis pigmentosa in a large kindred. J Med Genet 1991; 28: 453-457.

10 Musarella MA, A nson-Cartwright L, Leal SM et al: Multipoint linkage analysis and heterogeneity testing in twenty $\mathrm{X}$-linked retinitis pigmentosa families. $\mathrm{G}$ enomics 1990; 8: 286-296.

$11 \mathrm{D}$ ahl N, Sundvall $M$, Pettersson $U$ et al: Genetic mapping for loci of X linked retinitis pigmentosa. Clin G enet 1991; 40: 435-440.

12 M eire F M, Bergen A A B, D e R ouck A, L eys M, D elleman $\mathrm{JW}$ : X-linked progressive cone dystrophy: localisation of the gene locus to X p21-p11.1 by linkage analysis. Br J O phthalmol 1994; 78: 103-108.

13 R osenfeld PJ, McKusick VA, A mberger J S, D ryja TP: Recent advances in the gene map of inherited eye disorders: primary hereditary diseases of the retina, choroid, and vitreous. J M ed G enet 1994; 31: 903-915.

$14 \mathrm{H}$ egde A N, Inokuchi K, Pei W et al: U biquitin C-terminal hydrolase is an immediate-early gene essential for longterm facilitation in A plysia. Cell 1997; 89: 115-126.

15 L ubs H A , C hiurazzi P, A rena J F, Schwartz C, Tranebjaerg L, Neri G : X L M R genes: update 1996. A m J Med Genet 1996; 64: 147-157.

16 A Idred MA, Dry KL, Knight-Jones EB et al: Genetic analysis of a kindred with $\mathrm{X}$-linked mental handicap and retinitis pigmentosa. Am J H um Genet 1994; 55: 916-922.

17 Derry JMD, Jess U, Francke U : Cloning and characterization of a novel zinc finger gene in X p11.2. Genomics 1995; 30: 361-365.

18 Zehetner G, L ehrach H : The R eference Library system sharing biological material and experimental data. $\mathrm{N}$ ature 1994; 367: 489-491.
19 Berger W, M eindl A, de L eeuw B et al: Generation and characterization of radiation induced cell hybrids and isolation of probes from the proximal short arm of the human X chromosome. H um G enet 1992; 90: 243-246.

20 D ib C, Faure S, Fizames $C$ et al: A comprehensive genetic map of the human genome based on 5.264 microsatellites. Nature 1996; 380: 152-154.

21 Dear S, Staden R: A sequence assembly and editing program for efficient management of large projects. Nucleic A cids Res 1991; 19: 3907-3911.

$22 \mathrm{H}$ ayashi K: PCR -SSCP: a simple and sensitive method for detection of mutations in the genomic DNA. PCR Methods A ppl 1991; 1: 34-38.

23 Meindl A, D ry KL, Herrmann $K$ et al: A gene (RPGR) with homology to the R CCl guanine nucleotide exchange factor is mutated in X-linked retinitis pigmentosa ( $R P 3$ ). Nature G enet 1996; 13: 35-42.

24 Coleman M P, A mbrose HJ, Carrel L, N emeth A H, Willard $H F$, Davies KE: A novel gene, DXS8237E, lies within $20 \mathrm{~kb}$ upstream of U BE 1 in X p11.23 and has a different X inactivation status. G enomics 1996; 31: 135-138.

25 Thiselton DL, Hampson RM, Nayudu M et al: Mapping the RP2 locus for $X$-linked retinitis pigmentosa on proximal Xp: a genetically defined 5-cM critical region and exclusion of candidate genes by physical mapping. G enome Research 1996; 6: 1093-1112.

26 Coleman M P, Nemeth A H, Campbell L, R aut CP, Weissenbach J, Davies KE: A 1.8 M b YAC contig in Xp11.23: I dentification of $\mathrm{CpG}$ islands and physical mapping of CA repeats in a region of high gene density. G enomics 1994; 21: 337-343.

27 Schindelhauer D, Hellebrand H, Grimm L et al: Long range map of a 3.5-M b region in X p11.23 with a sequence ready map from a $1.1 \mathrm{Mb}$ gene-rich interval. Genome Research 1996; 6: 1056-1069. 